Methods CARTaGENE is the largest prospective cohort in Quebec with 43,000 participants recruited among the general population aged 40-69 years at baseline. Approximately 10,000 participants filled out an occupational history questionnaire and data were then coded to create a job titles and industry types database. Then, the CANJEM matrix was applied to assign exposure to 258 chemical agents based on occupations (probability and median dose of exposure).

Results The 10,895 CARTaGENE participants reported a total of 21,612 jobs, $45 \%$ were held by men and $55 \%$ by women. For 1,253 jobs $(5.8 \%)$ occupation code in the NOC 2011 system was impossible to assign because of lacking information. The majority of jobs were in white collar occupations (18.97\%). Among the most prevalent exposures $(>10 \%$ jobs having probability of exposure $>25 \%)$ in the cohort were solvents, polycyclic aromatic hydrocarbons, cleaning agents, biocides, engine emissions and aliphatic alcohols. Overall, 18 agents have an overall prevalence greater than 5\%, while a further 64 agents have a prevalence greater that $1 \%$.

Conclusion Such data is relevant from a public health perspective that uses a population-based approach. CARTaGENE has the advantage to integrate a rich collection of data on each participant such as health questionnaires (diseases, lifestyle), physical measures (blood pressure, spirometry), biochemical data (triglyceride, creatinine), genetic data that could be combined to occupational history data. Ultimately, this public resource available to researchers worldwide allows to carry out further research on specific diseases or exposures conditions.

\section{RF-421 KEYWORD SEARCHES OF 0*NET JOB TASKS FOR EXPOSURE ASSESSMENT, UNITED STATES OF AMERICA}

${ }^{1}$ John Beard, Mitchell Greenhalgh, Emily Hawkes, Bailey Jones, Sarah Hamner, Steven Thygerson. 'Brigham Young University, United States

\subsection{6/OEM-2021-EPI.374}

Introduction Job exposure matrices are often used for exposure assessment in occupational exposure and epidemiology studies. However, general population job exposure matrices are difficult to find and access for workers in the United States of America (U.S.).

Objectives We aimed to use publicly available information to determine exposure to a wide range of occupational agents for use in U.S. general population studies.

Methods We used information from the U.S. Department of Labor's Occupation Information Network database (O*NET) for 19,636 job tasks and 974 civilian occupations. We used automated keyword searches of each job task to identify job tasks that involved exposure to 50 occupational agents. We had two reviewers determine whether each identified job task actually involved exposure to the 50 occupational agents. We calculated percent agreement to compare the reviewers' exposure determinations for each job task and exposure. We had a third reviewer, a certified industrial hygienist (CIH), assess any job task and exposure for which the two reviewers disagreed. The third reviewer also assessed a 10\% sample of job tasks and exposures for which the two reviewers agreed. For each occupation, we used this information to derive three exposure variables for each occupational agent: ever exposure, number of job tasks of exposure, and frequency of exposure.

Results Our keyword searches identified a median of nine (interquartile range: 2.0, 40.5) job tasks for each occupational agent and the maximum was 308. The median percent agreement for the two reviewers' exposure determinations was 95\% (interquartile range: 79\%, 100\%). The median percentage for ever exposure to the occupational agents was $0.41 \%$ (interquartile range: $0.10 \%, 1.08 \%$ ) and the maximum was $14.48 \%$.

Conclusion O*NET information can be used to determine exposure to a wide range of occupational agents. We intend to use O*NET information in epidemiological studies of the U.S. general population.

\section{RF-449 THE RATE OF NOISE-INDUCED HEARING LOSS IN RELATION TO OCCUPATIONAL NOISE EXPOSURE AMONG WORKERS IN ISRAEL}

${ }^{1}$ Nyasha Makaruse, Ora Paltiel, Lilah Rinsky Halivni. ${ }^{1}$ Hebrew University of Jerusalem, Israel

\subsection{6/OEM-2021-EPI.375}

Introduction Annual audiometric screening is the default protocol for occupational settings. This frequency of testing is costly for the health system, employees and employers in terms of money, resources and time.

Objectives To investigate the annual rate of clinical deterioration of hearing of workers. Secondly, the study aimed to establish an effective, efficient, hearing screening frequency protocol for noise induced hearing loss (NIHL) screening in noise exposed workers in Israel.

Methods A historical cohort study was conducted. Audiometric surveillance data from the Jerusalem occupational medicine registry of employees in various industries across the district from 2006-2017 were used. Plants from Jerusalem district with noise levels, equal to or greater than a timeweighted average (TWA) sound level of $85 \mathrm{~dB}(\mathrm{~A})$ for 8 -hour work day were considered hazardous. Joinpoint regression was used to assess significant changes in the trend of detected hearing loss for different time intervals between audiological check-ups.

Results 263 noise exposed workers and 93 reference workers were followed for 12 years producing 1913 audiograms for analyses. Threshold shifts among noise-exposed workers became significantly stable at approximately $1 \mathrm{~dB} /$ year at three years $(p=0.037)$ using frequencies $1-4 \mathrm{kHz}$. Noise- exposed workers, aged below 50 at entry, showed stabilized threshold shifts of $0.8 \mathrm{~dB}$ annually on intervals of 3-8 years, compared to $0.35 \mathrm{~dB}$ shift annually using the $0.5-2 \mathrm{kHz}$.

Conclusions The trend of mean hearing threshold shifts in noise-exposed workers becomes a nearly constant at $0.8 \mathrm{~dB}$ shift annually at 3 years onwards. These small annual changes are within the measurement error of audiometers $( \pm 5 \mathrm{~dB})$. Therefore, intervals between subsequent tests should be considered so as to identify a significant and real deterioration in hearing and avoid unnecessary medical investigations. A triennial audiometric screening frequency would be a better surveillance method for noise exposed workers in the category of $85-90 \mathrm{~dB}(\mathrm{~A})$ without other known risk factors. 